\title{
Nafion Modified Graphene Aerogel with Hierarchical Porous Structures
}

\author{
WANG Yong ${ }^{1,2}$, YU Yun ${ }^{1}$, FENG Ai-Hu ${ }^{1,2}$, JIANG Feng ${ }^{1,2}$, HU Xue-Bing ${ }^{3}$, SONG Li-Xin ${ }^{1}$ \\ (1. Chinese Academy of Sciences Key Laboratory of Inorganic Coating Materials, Shanghai Institute of Ceramics, Chinese \\ Academy of Sciences, Shanghai 200050, China; 2. University of Chinese Academy of Sciences, Beijing 100049, China; 3. Key \\ Laboratory of Inorganic Membrane, Jindezhen Ceramic Institute, Jingdezhen 333001, China)
}

\begin{abstract}
A novel graphene aerogel with pore size less than $1 \mu \mathrm{m}$ was synthesized by Sol-Gel method, in which Nafion was skillfully introduced as modifiers connecting graphene oxides (GO). Ethylenediamine was used to produce a chemically linked graphene hydrogel during reducing process, which can then be freeze-dried to remove the absorbed water to form graphene aerogel. Nafion in GO solution prohibits the re-stacking of individual graphene sheets during reduction and freeze-drying process. By the above method, the pore size of as-prepared graphene aerogel could be effectively controlled within $1 \mu \mathrm{m}$, much less than that of the traditional graphene aerogel (20$100 \mu \mathrm{m})$. The new graphene aerogel with unique structure exhibited excellent properties, such as high specific surface area, high porosity, and better electrochemical properties. All data suggest that this graphene aerogel may have promising application prospect in the field of energy.
\end{abstract}

Key words: three-dimensional graphene; graphene aerogel; graphene; graphene oxide; Nafion

Graphene is a two-dimensional material composed of only one layer carbon atoms, which are $\mathrm{sp}^{2}$-bonded in a planar hexagonal structure ${ }^{[1]}$. This special structure endorses graphene many outstanding properties, such as good transparency ${ }^{[2]}$, large specific surface area ${ }^{[3]}$, great thermal $^{[4-5]}$ and electrical conductivity ${ }^{[6]}$, and good mechanical properties and chemical stability ${ }^{[7]}$. Graphene has been widely used in the area of composite materials ${ }^{[8]}$, energy ${ }^{[9]}$ and other fields for these properties.

Although graphene is stable once prepared, the planar structure introduces strong Van der Waals forces when the distance of different graphene sheets is close enough. The outstanding properties of single layer graphene would be seriously damaged when this nano material was agglomerated. It is difficult to reverse the agglomeration because of the strong $\pi-\pi$ interaction between graphene layers $^{[10]}$. Thus, the most important challenge in the research and application of graphene is maintaining graphene in single layer state. Graphene aerogel is a graphene derivative with three dimensional porous structures. This structure effectively constrains the agglomeration of graphene and extend the application of planar graphene to more areas like Li battery ${ }^{[11]}$ and supercapacitors $^{[9]}$.

The preparation of graphene aerogel is generally divided into two categories, direct fabrication with pristine graphene through template methods ${ }^{[12]}$, or the self-assembling of graphene oxide (GO) dispersion ${ }^{[13]}$. Fabrication with pristine graphene was limited by special template materials and CVD equipments ${ }^{[13]}$. Fabricating graphene aerogel through GO way was more favorable, as it can be prepared in a large scale through liquid phase reaction. The oxygen containing groups $(-\mathrm{OH},-\mathrm{O}-$, $-\mathrm{COOH}$ ) connected on the basal plane of GO make it possible to be dispersed in water stably without other surfactants. Also GOcould be restored to planar hexagonal structure by the reduction with different agents ${ }^{[14]}$. However, it is still difficult to prepare graphene aerogel with micropores with GO. The preparation of graphene aerogels with small pore size usually needs special equipment. It has been reported that graphene aerogel with pore size about $5 \mu \mathrm{m}$ was prepared through supercritical drying $^{[15]}$ and pore size $10-20 \mu \mathrm{m}$ was prepared by liquid nitrogen freezing ${ }^{[16]}$.

In this study, the preparationof graphene aerogels by self-assembling of GO nano sheets was improved by premodifying GO with a surfactant Nafion. The graphene aerogel fabricated through this method had a multi-scale porous structure with macropores of $50-100 \mu \mathrm{m}$, and micropores about $1 \mu \mathrm{m}$. Graphene aerogel with this hierarchical porous structure was found had high specific surface area and better electrochemical performance. 


\section{Experiments}

\subsection{Materials and reagents}

Graphite powder (5000 mesh), ethylenediamine (EDA; AR), Nafion (5wt\%; D520) were obtained from SigmaAldrich and used as received. Potassium permanganate $\left(\mathrm{KMnO}_{4}\right.$; GR), hydrogen peroxide $\left(\mathrm{H}_{2} \mathrm{O}_{2} ; 30 \%\right.$, AR), concentrated sulfuric acid $\left(\mathrm{H}_{2} \mathrm{SO}_{4} ; 98 \%\right.$, AR), hydrogen chloride $(\mathrm{HCl} ; 37 \%$; $\mathrm{AR})$, sodium nitrate $\left(\mathrm{NaNO}_{3}\right.$; $\left.\mathrm{AR}\right)$ were purchased from Sinopharm Chemical Reagent Co., Ltd.

\subsection{Preparation of graphene aerogel}

GO was fabricated by the oxidation of flake graphite according to the modified Hummers methods. In a typical procedure, $1 \mathrm{~g}$ flake graphite and $0.5 \mathrm{~g} \mathrm{NaNO}_{3}$ were dispersed in $75 \mathrm{~mL} \mathrm{H}_{2} \mathrm{SO}_{4}$ by stirring in an ice-water bath. $6 \mathrm{~g} \mathrm{KMnO}_{4}$ was added slowly into the dispersion in small portions within 30 min to maintain the temperature under $10{ }^{\circ} \mathrm{C}$. Then, the dispersion was magnetically stirred for additional $4 \mathrm{~min}$ in the ice-water bath, followed by stirring at room temperature for $12 \mathrm{~min}$ and $70^{\circ} \mathrm{C}$ for 2 h. $100 \mathrm{~mL}$ deionized water was added slowly followed by stirring at $90^{\circ} \mathrm{C}$ for additional $15 \mathrm{~min} .100 \mathrm{~mL}$ deionized water was added to terminate the reaction and $5 \mathrm{~mL} \mathrm{H}_{2} \mathrm{O}_{2}$ was added to remove excessive oxidant. After cooling, the mixture was purified with $3 \% \mathrm{HCl}$ solution for 3 times, and deionized water 5 times to $\mathrm{pH}=7$ by centrifugation. Finally, the solution was sonicated for $60 \mathrm{~min}$ and centrifuged at $5000 \mathrm{r} / \mathrm{min}$ for $10 \mathrm{~min}$ to collect the supernatant. The resulting GO dispersion was prepared by diluting the supernatant to $2 \mathrm{mg} / \mathrm{mL}$.

$0.5 \mathrm{~mL} 5 \mathrm{wt} \%$ Nafion solution was added to $50 \mathrm{~mL} \mathrm{GO}$ dispersion, followed by sonicated for $30 \mathrm{~min}$. The dispersion was magnetically stirred for $2 \mathrm{~h}$ before $0.5 \mathrm{~mL}$ EDA was added. Then the dispersion was placed in a sealed vessel and heated at $90^{\circ} \mathrm{C}$ for $24 \mathrm{~h}$. The hydrogel formed during the heating was washed by merging it in deionized water for one week with water changed every 6 h. Finally, graphene aerogel was prepared by the freezedrying of the hydrogel at $-60^{\circ} \mathrm{C}$. The sample prepared was denoted as GA-N. In a controlled experiment, graphene aerogel was prepared without Nafion modification, and this sample was denoted as GA. Hydrazine hydrate reduced GO was denoted as rGO in this experiment.

\subsection{Characterizations}

Atomic force microscopy (AFM) study was performed to measure the thickness of GO using AFM prober (Bruker Dimension Icon, Bruker, Germany) in the ScanAsyst mode. The samples were prepared by dropping diluted aqueous dispersion of $\mathrm{GO}(0.04 \mathrm{mg} / \mathrm{mL})$ on freshly cleaved mica and dried at $80^{\circ} \mathrm{C}$. The surface morphologies were characterized with a scanning electron microscope (SEM; S3400N, Hitachi, Japan) after coated with gold using a sputter coater. The sample of graphene adsorbed with Nafion was prepared with freeze-drying without washing. The surface X-ray diffraction (XRD) patterns were measured with X-ray diffractometer (D8 Advance, Bruker, Germany) using a Cu-K X-ray source $(0.15418 \mathrm{~nm})$. The organic groups were measured with Fourier transformed infrared spectroscopy (FT-IR) spectrometer (NICOLET Is10, Thermo, USA) in room temperature using the $\mathrm{KBr}$ pellet technique. The structure features of the samples were acquired by confocal Raman microscope (Thermo Nicoet, USA) using powder form samples. In addition, the specific area of the samples was measured by a BET surface area analyzer (ASAP2010, Micrometrics, USA). The samples were heated at $300^{\circ} \mathrm{C}$ under vacuum for more than $4 \mathrm{~h}$ before BET measurements. To perform CV test, porous carbon material was mixed with acetylene black and polytetrafluoroethylene at a mass ratio of $80: 10: 10$, then ethanol was added to makea slurry.Working electrode was prepared by uniformly pasting the slurry on $10 \mathrm{~mm} \times 10 \mathrm{~mm}$ nickel foamand dryingat $90^{\circ} \mathrm{C}$ for $2 \mathrm{~h}$. The cyclic voltammetric characteristics of each sample were determined using a three-electrode system with Solartron-1287 electrochemical workstation with scanning rate at $10 \mathrm{mV} / \mathrm{s}$ in a $1 \mathrm{~mol} / \mathrm{L} \mathrm{KCl}$ aqueous solution.

\section{Results and discussion}

\subsection{Characteristics}

Fig. 1 shows the AFM images of GO before and after Nafion modification. The thickness of these two-dimensional materials and their surface morphology were clearly illustrated in the figure. It can be seen the surface of GO is smooth and flat before Nafion modification. The particle size of GO was about 400 nm (Fig. 1(a)), and the thickness was about $1 \mathrm{~nm}$ (Fig. 1(c)). When GO was modified with Nafion, a large number of small particles were found evenly distributed on the nano sheets even after the long-time sonication (Fig. 1(b)). Although some particles appeared on the mica substrate, it can be seen that these particles tend to be adsorbed onto the surface of GO. The height of these small molecular particles is $2-3 \mathrm{~nm}$ (Fig. 1(d)). Agglomeration is not observed between the layers of the GO sheets, which suggests that a small amount of Nafion can effectively modify the surface of GO without damaging its dispersion.

Fig. 2 is XRD patterns of graphene, flake graphite, rGO, and GA and GA-Nafion powders. (002) characteristic peaks of GO and flake graphite are $11.85^{\circ}$ and $25.74^{\circ}$ respectively, corresponding to the inter-layer space $0.75 \mathrm{~nm}$ and $0.35 \mathrm{~nm}$. The inter-layer space increase from graphite to GO was attributed to the oxygen- containing functional groups on carbon layers. These functional 



Fig. 1 AFM height image of GO (a), GO modified with Nafion (b) and the height profiles of GO (c) and GO modified with Nafion (d)

groups facilitated the peeling of carbon layer from densely packed graphite. The characteristic peak disappeared at rGO, which indicates the restoration graphene plane. The aerogel prepared by GO with or without Nafion modification had similar peaks at $13.37^{\circ}$ and $13.17^{\circ}$ respectively, representing the inter-planar space of $0.66 \mathrm{~nm}$ and $0.67 \mathrm{~nm}$. The characteristic peaks of these two materials show that the oxygen-containing functional groups were not completely removed by EDA reduction.

Similar results were also found in Raman spectroscopy. The structure of graphene related materials can be characterized with Raman spectroscopy. Significant peaks denoted as $\mathrm{D}$ and $\mathrm{G}$ peaks appeared on all the spectra (Fig. 3). The D peak is related to the oscillation of the $\mathrm{sp}^{2}$ carbon bond, but this peak is only activated when there are defects nearby. Therefore, D peak can be used to evaluate the defect density of graphene related materials.

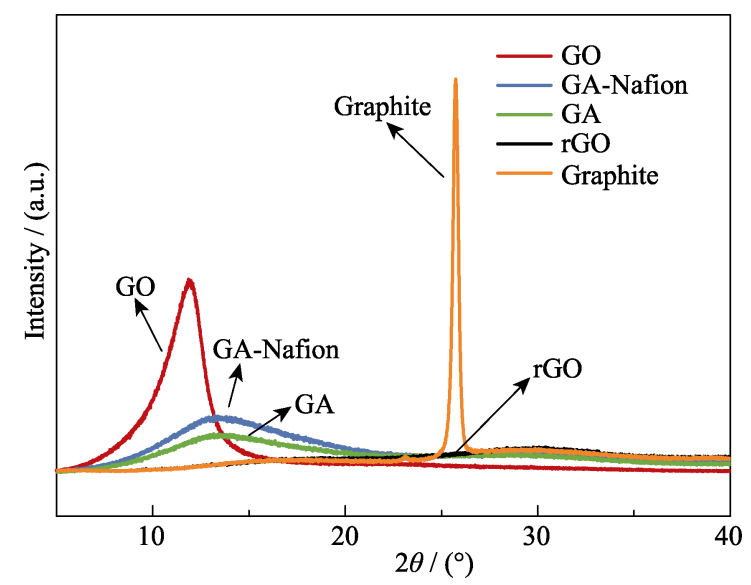

Fig. 2 XRD patterns of GO, rGO, graphite, GA and GA-Nafion
And the $\mathrm{G}$ peak comes from the first-order scattering of the $E_{2 \mathrm{~g}}$ mode of $\mathrm{sp}^{2}$ domain from the stretching vibration of in-plane $\mathrm{C}-\mathrm{C}$ bonds ${ }^{[17]}$. The intensity ratio $I_{\mathrm{D}} / I_{\mathrm{G}}$ is generally used to quantify the defect density of graphene related materials. $I_{\mathrm{D}} / I_{\mathrm{G}}$ of GO, GA, GA-Nafion and rGO was $1.13,1.22,1.24$ and 1.46 respectively. It can be observed that the ratio increased when GO was reduced. Therefore, the removal of oxygen-containing functional groups would leave defects on graphene plane. The ratios of graphene aerogel prepared by GO with or without Nafion modification are very close, indicating that the modification of Nafion does not affect the reaction between graphene and EDA. The high D/G intensity ratio of rGO may be a result of its higher reduction level.

It can be seen more clearly in the FT-IR spectra that there were not significant differences in the composition of these aerogels fabricated through different routes (Fig. 4). The FT-IR spectra of GO shows characteristic peaks of the stretching vibration of hydroxyl group at $3435 \mathrm{~cm}^{-1}$, $\mathrm{C}==\mathrm{C}$ stretching vibrations of benzene rings at $1626 \mathrm{~cm}^{-1}$, $\mathrm{C}==\mathrm{O}$ stretching vibrations of carboxylic groups at $1716 \mathrm{~cm}^{-1}$, $\mathrm{C}-\mathrm{OH}$ stretching vibrations at $1395 \mathrm{~cm}^{-1}$ and $\mathrm{C}-\mathrm{O}$ stretching vibrations of epoxide group at $1044 \mathrm{~cm}^{-1}$. The

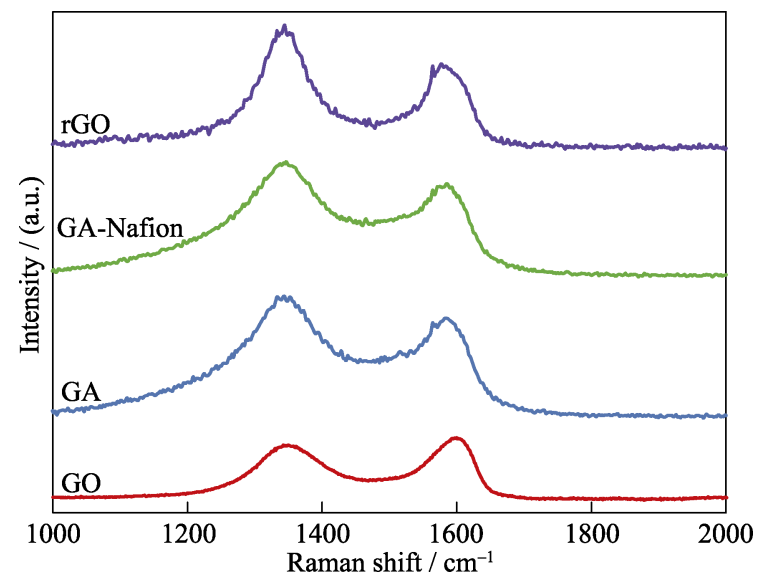

Fig. 3 Raman spectra of GO, rGO, GA and GA-Nafion

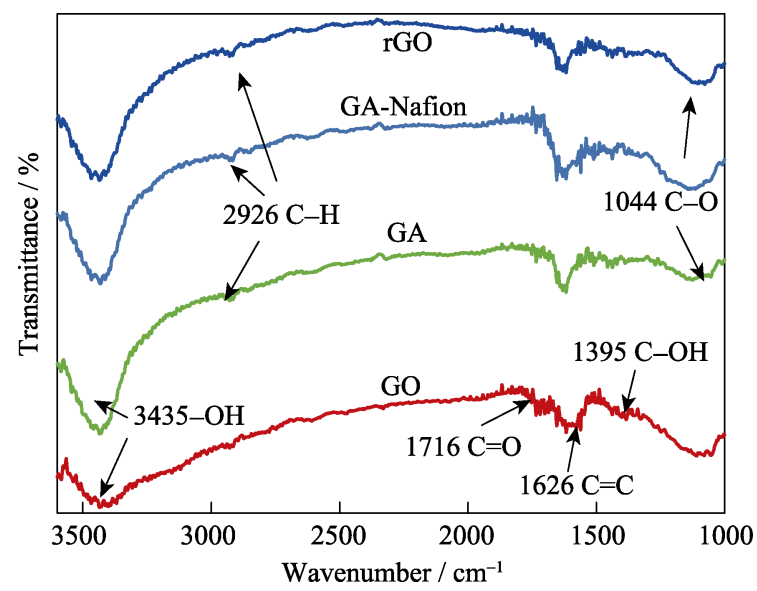

Fig. 4 FT-IR spectra of GO, rGO, GA and GA-Nafion 
peaks of hydroxyl and epoxide groups in rGO, GA and GA-Nafion were weakened because of the reduction by EDA. At the same time, the GA-Nafion shows almost the same spectrum as GO. Characteristic peaks related to the $\mathrm{F}$ atom are not observed after a long time washing, indicating that Nafion adsorbed on the GO nano sheets was completely removed.

\subsection{Morphologies}

It has been demonstrated that, GA and GA-Nafion has similar composition, but the morphologies were quite different. Fig. 5(a) shows the morphology of GA, which has typical porous structures with pore size of $20-50 \mu \mathrm{m}$. It is this porous structure that prevented the agglomeration of graphene, and promoted the performance of graphene aerogel in energy and environment area. Fig. 5(b) is morphologies of GO with Nafion adsorbed on the surface. NumerousNafion nano fibers existed between multi-layer stacked GO sheetthough only small amount of Nafion was added. It was clearly demonstrated in Fig. 5(c) and (d) that, the structure of graphene aerogel was significantly changed when GO nano sheets were pre-modified with Nafion. GA-Nafion had a multi-scale porous structure, with macropores about $100 \mu \mathrm{m}$, and micropores about $1 \mu \mathrm{m}$. As graphene aerogel was freeze-dried during the preparation, such macropores were likely formed during the crystallization of ice, and the micropores were formed because of the absorbed Nafion molecules.

Fig. 6 is the structure of Nafion molecule which has long hydrophobic fluorocarbon chains and hydrophilic sulfonic groups ${ }^{[18]}$. When Nafion was added into GO dispersion, the sulfonic groups formed hydrogen bonds with carboxyl and epoxy groups of GO. And the off-plane fluorocarbon chains could prohibit the stacking GO nano sheets. Also, Nafion would form micelles with hydrophobic cores and hydrophilic surfaces when it is distributed in water because of its bipolar structure. These micelles became soft templates to form micropores with GO attached on the surfaces. The soft template effect and prohibited agglomeration lead to the formation of micropores. The multi scale porous structure was a combination of macropores from ice and micropores from Nafion.

\subsection{Performance}

The different performance of two kinds of graphene aerogels was demonstrated in the BET specific surface area and electrochemical properties. As shown in Fig. 7, the specific surface area of the powder obtained by the direct drying of GO was only $32.8 \mathrm{~m}^{2} / \mathrm{g}$, and the specific surface area of rGO after freeze-drying was increased to $437.4 \mathrm{~m}^{2} / \mathrm{g}$. GA has even higher specific surface area, which came from the network formed between EDA and GO sheets (763.9 $\left.\mathrm{m}^{2} / \mathrm{g}\right)$. The specific surface area of GANafion was increased to $967.8 \mathrm{~m}^{2} / \mathrm{g}$, which was more close to the theoretical area of graphene material ${ }^{[2]}$. The result demonstrated that the multi-scale porous structure of aerogel improved the specific surface area. This material may have great application prospects in the application areas that require relatively high specific surface area. Fig. 8 shows the electrochemical cyclic voltammetry curves of activated carbon, GA and GA-Nafion. The specific capacities of AC, GA and GA-Nafion calculated from the figure were 51.7, 103.4, and $146.1 \mathrm{~F} / \mathrm{g}$, repectively. GA has better electrochemical performance than activated carbon, which may be attributed to its high
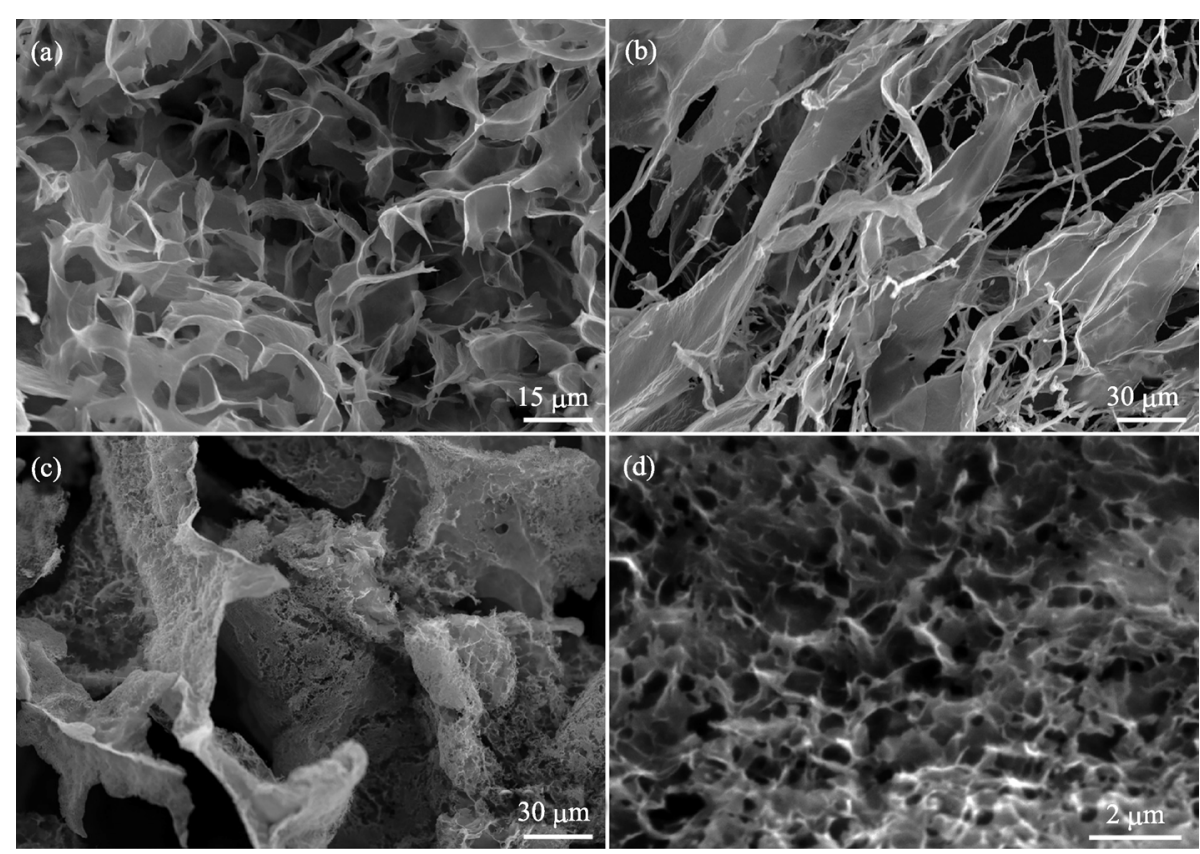

Fig. 5 SEM morphologies of GA (a), GO nano sheets modified with Nafion (b), GA-Nafion (c) and its high resolution morphology (d) 


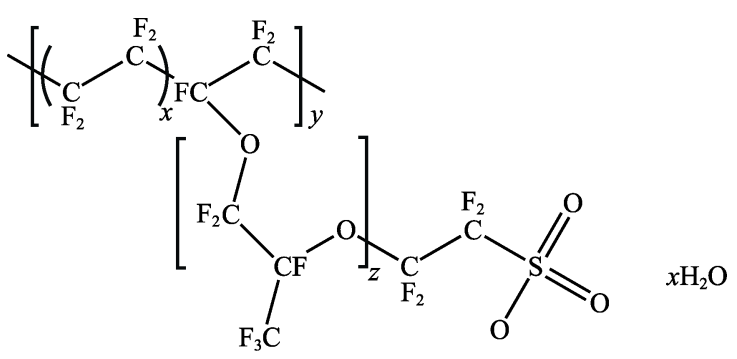

Fig. 6 Chemical structure of Nafion

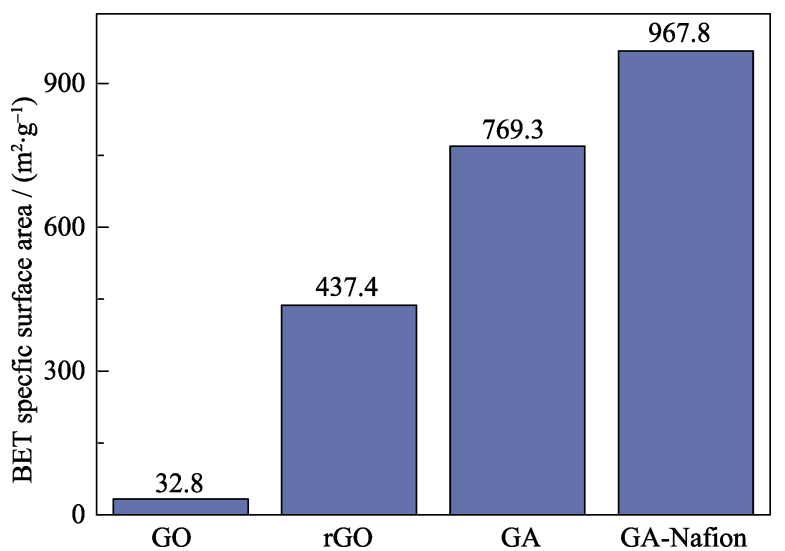

Fig. 7 Specific surface area of GO, rGO, GO-EDA, and GA-Nafion

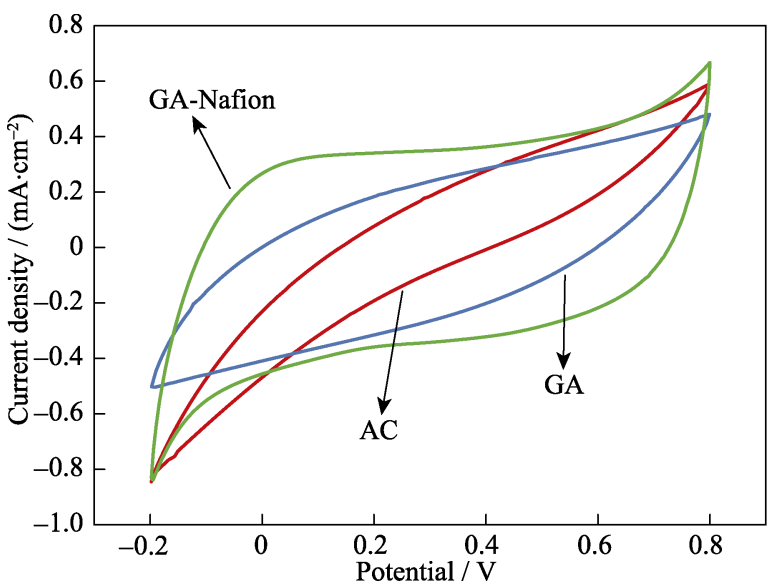

Fig. 8 Cyclic voltammogram of activated carbon (AC), GA and GA-Nafion

surface area. The performance of multi- scale porous GA-Nafion was enhanced more significantly. This indicates that graphene aerogel prepared through Nafion modified GO has better performance for its specicial porous sturcture.

\section{Conclusions}

This study demonstrates that Nafion can act as a surfactant to modify the surface of GO nano sheets. And the graphene aerogel prepared through Sol-Gel methods with this modified GO sheets exhibits multi-scale porous structures, with macropores $50-100 \mu \mathrm{m}$ in size, and micro- pores about $1 \mu \mathrm{m}$. This special graphene aerogel has large specific surface areas close to $967.8 \mathrm{~m}^{2} / \mathrm{g}$, showing better electrochemical performance over traditional graphene aerogel.

\section{References}

[1] ALLEN M J, TUNG V C, KANER R B. Honeycomb carbon: a review of graphene. Chemical Reviews, 2010, 110(1): 132-145.

[2] NAIR R R, BLAKE P, GRIGORENKO A N, et al. Fine structure constant defines visual transparency of graphene. Science, 2008, 320(5881): 1308-1308.

[3] CHEN H, GAO H, XIAO H W, et al. Eco-friendly synthesis of few-layer graphene with high surface area under low temperature for supercapacitors. Electrochimica Acta, 2016, 206: 10-16.

[4] OH J, YOO H, CHOI J, et al. Significantly reduced thermal conductivity and enhanced thermoelectric properties of single- and bi-layer graphene nanomeshes with sub-10 nm neck-width. Nano Energy, 2017, 35: 26-35.

[5] BALANDIN A A, GHOSH S, BAO W, et al. Superior thermal conductivity of single-layer graphene. Nano Letters, 2008, 8(3): 902-907.

[6] JO Y K, KIM I Y, KIM S J, et al. Non-monotonous dependence of the electrical conductivity and chemical stability of a graphene freestanding film on the degree of reduction. RSC Advances, 2015, 5(20): 19259-19263.

[7] PALERMO V, KINLOCH I A, LIGI S, et al. Nanoscale mechanics of graphene and graphene oxide in composites: a scientific and technological perspective. Advanced Materials, 2016, 28(29): 6232-6238.

[8] DU J, CHENG H M. The fabrication, properties, and uses of graphene/polymer composites. Macromolecular Chemistry and Physics, 2012, 213(10/11): 1060-1077.

[9] ZHU C, LIU T, QIAN F, et al. Supercapacitors based on three-dimensional hierarchical graphene aerogels with periodic macropores. Nano Letters, 2016, 16(6): 3448-3456.

[10] WANG J, CHEN B, XING B. Wrinkles and folds of activated graphene nanosheets as fast and efficient adsorptive sites for hydrophobic organic contaminants. Environmental Science \& Technology, 2016, 50(7): 3798-3808.

[11] AHN W, SEO M H, JUN Y S, et al. Sulfur nanogranular film-coated three-dimensional graphene sponge-based high power lithium sulfur battery. ACS Applied Materials \& Interfaces, 2016, 8(3): 1984-1991.

[12] SHI J L, TANG C, PENG H J, et al. 3D mesoporous graphene: CVD self-assembly on porous oxide templates and applications in high-stable Li-S batteries. Small, 2015, 11(39): 5243-5252.

[13] KOTAL M, KIM J, OH J, et al. Recent progress in multifunctional graphene aerogels. Frontiers in Materials, 2016, 3: 29.

[14] DREYER D R, PARK S, BIELAWSKI C W, et al. The chemistry of graphene oxide. Chemical Society Reviews, 2010, 39(1): 228-240.

[15] ZHANG X, SUI Z, BIN X, et al. Mechanically strong and highly conductive graphene aerogel and its use as electrodes for electrochemical power sources. Journal of Materials Chemistry, 2011, 21(18): 6494-6497.

[16] XIE X, ZHOU Y, BI H, et al. Large-range control of the microstructures and properties of three-dimensional porous graphene. Scientific Reports, 2013, 3: 2117.

[17] KRISHNAMOORTHY K, VEERAPANDIAN M, MOHAN R, et al. Investigation of Raman and photoluminescence studies of reduced graphene oxide sheets. Applied Physics A: Materials Science \& Processing, 2012, 106(3): 501-506.

[18] SHI W, BAKER L A. Imaging heterogeneity and transport of degraded Nafion membranes. RSC Advances, 2015, 5(120): 9928499290. 


\section{Nafion 改性多级孔径石墨烯气凝胶制备与性能研究}

王 勇 ${ }^{1,2}$, 于 云 ${ }^{1}$, 冯爱虎 ${ }^{1,2}$, 江 峰 ${ }^{1,2}$, 胡学兵 ${ }^{3}$, 宋力昕 ${ }^{1}$

(1. 中国科学院 上海硅酸盐研究所无机涂层重点实验室, 上海 200050; 2. 中国科学院大学, 北京 100049; 3. 景德 镇陶瓷大学 江西省高等学校无机膜重点实验室, 景德镇 333001)

摘 要: 通过溶胶-凝胶法制备了孔径小于 $1 \mu \mathrm{m}$ 的多级孔径新型石墨烯气凝胶。制备过程中, 首先通过 Nafion 对氧 化石墨烯(GO)表面进行化学修饰, 并利用乙二胺还原制备石墨烯水凝胶, 最终通过冷冻干燥形成石墨烯气凝胶。实 验发现 Nafion 可以有效减少制备过程中氧化石墨烯的团聚，使石墨烯气凝胶形成多级孔径形貌。所得石墨烯气凝 胶的孔径可控制在 $1 \mu \mathrm{m}$ 以内，远小于传统石墨烯气凝胶材料的孔径 $(20 \sim 100 \mu \mathrm{m})$ 。这种具有独特结构的石墨烯气凝 胶表现出优异的性能，例如高比表面积，高孔隙率，其电化学电容性能相对传统气凝胶提高了约 40\%。

关 键 词: 三维石墨烯; 石墨烯气凝胶; 石墨烯; 氧化石墨烯; Nafion

中图分类号: 0613 文献标识码: A 